\title{
ELETROMIOGRAFIA DOS MÚSCULOS DO CORE EM DIFERENTES EXERCÍCIOS DE ESTABILIZAÇÃO DO TRONCO
}

\author{
F. C. Gregorio*, C. E. D. S. Pereira, **, F. J. Arantes*, F. H. D. O. Silva**, J. B. Destro-Filho***, \\ F. B. Lizardo**** \\ *Programa Pós-Graduação Engenharia Biomédica UFU, Uberlândia, Brasil \\ **Faculdade de Educação Física UFU, Uberlândia, Brasil \\ ***Docente de pós-graduação em engenharia biomédica PPGEB - UFU, Uberlândia, Brasil \\ ****Docente do Instituto de Ciências Biomédicas ICBIM - UFU, Uberlândia, Brasil \\ e-mail: fabiocgregorio@hotmail.com
}

\begin{abstract}
Resumo: o core está relacionado com a região do tronco e possui como característica estabilizar o tronco durante o movimento dos membros. O objetivo foi avaliar o sinal eletromiográfico dos músculos do core em sete exercícios de estabilização de tronco. A amostra foi composta por dez voluntários do gênero masculino. Os resultados mostraram que os padrões de ativação muscular caracterizam-se pela ativação preferencial dos músculos que atuam contra a gravidade. Os exercícios de estabilização de tronco podem ser utilizados para desenvolver a resistência muscular e a estabilidade da coluna vertebral.
\end{abstract}

Palavras-chave: músculos abdominais, prancha ventral, prancha lateral, ponte

Abstract: the core is related to the region of the torso
and has as a characteristic to stabilize the trunk during
the movement of the limbs. The objective was to
evaluate the electromyographic signal the muscles of
the core into seven stabilization exercises. The sample
was composed of ten male volunteers. The results
showed that muscle activation patterns are
characterized by preferential activation of the muscles
that act against gravity. Trunk stabilization exercises
can be used to develop muscular endurance and
stability of vertebral column. Keywords: abdominal muscles, ventral, lateral plank, plank bridge

\section{Introdução}

O core é um segmento do corpo relacionado com o tronco ou, mais especificamente, com a região lombarpélvica e sua estabilidade é fundamental para proporcionar uma base durante movimentos dos membros superior e inferior em atividades da vida diária ou gestos esportivos, bem como suportar cargas, prevenir disfunções como lombalgia, desenvolver força e proteger a medula espinal e suas raízes neurais [1].

Exercícios de estabilização do tronco são frequentemente utilizados em programas de prevenção/reabilitação de lombalgia ou treinamento esportivo e muitos destes consistem na manutenção da posição "neutra" da coluna com aplicação de diferentes tipos de sobrecarga, como movimento dos membros [2], utilização de superfícies instáveis ou a combinação dessas estratégias.

A eletromiografia de superfície é uma técnica não invasiva de avaliação da função muscular que compreende a somatória dos potenciais elétricos oriundos de unidades motoras ativas, detectados por meio de eletrodos posicionados na pele [3], o qual permite avaliar a eficácia dos exercícios de estabilização por meio da análise da intensidade da ativação e coordenação dos músculos do tronco [2].

Desta forma, existem lacunas na literatura sobre uma periodização dos exercícios de estabilização do tronco baseado nos achados eletromiográficos. Este estudo será realizado com finalidade de fornecer uma medida quantitativa da ativação muscular em diferentes exercícios de estabilização, evidenciando quais exercícios são mais eficazes no recrutamento da musculatura do core, proporcionando uma base teórica para o direcionamento de programas de prevenção, reabilitação e treinamento esportivo que objetivam o desenvolvimento e fortalecimento dos músculos do core.

Portanto, objetivou-se analisar a atividade eletromiográfica dos músculos reto do abdome (RA), oblíquo externo do abdome (OE), oblíquo interno do abdome (OI), eretor da espinha (EE) e multífido (MU) durante sete variações de exercícios isométricos de estabilização do tronco.

\section{Método}

Caracterização da pesquisa e amostra: trata-se de um estudo de caráter experimental-quantitativo, aprovado pelo Comitê de Ética em Pesquisa (número 174.012) da Universidade Federal de Uberlândia (UFU) e desenvolvido no Laboratório de Eletromiografia Cinesiológica (LABEC) da UFU.

A amostra foi composta por 10 voluntários do gênero masculino com idade 20,9 $\pm 1,62$ anos, massa corporal $65,5 \pm 11,0 \mathrm{~kg}$, estatura $1,71 \pm 0,10 \mathrm{~m}$ e Índice de Massa Corpórea 22,24 $\pm 2,50 \mathrm{~kg} / \mathrm{m} 2$. Como critérios de inclusão, todos os indivíduos deveriam ser praticantes de atividade física regular pelo menos três vezes por semana nos últimos três meses, incluindo 
exercícios aeróbios e treinamento resistido [4], ser considerados fisicamente ativo ou muito ativo, de acordo com a classificação do questionário internacional de atividade física (IPAQ versão curta), e possuir IMC normal.

Para o registro eletromiográfico, foi utilizado o eletromiógrafo computadorizado da MyosystemBr1 P84/DATAHOMINIS Tecnologia ${ }^{\circledR}$ (Uberlândia, MG, Brasil), projetado de acordo com normas da InternationalSocietyofElectrophysiologyandKinesiology (ISEK), o qual possui impedância de entrada de 1015 Ohms, conversor analógico/digital com resolução de 16 bits, filtros Butterworth e bateria recarregável integrada. O MyosystemBr1 P84 foi conectado a um laptop/notebook alimentado apenas por bateria. Os sinais eletromiográficos foram coletados e processados posteriormente usando um aplicativo de software Myosystem Br1 (versão 3.5.6). A frequência de amostragem utilizada foi de $2000 \mathrm{~Hz}$ por canal durante toda a coleta, e os sinais eletromiográficos foram submetidos a um filtro passa-alta de $20 \mathrm{~Hz}$ e passabaixa de $1000 \mathrm{~Hz}$.

Para a captação dos sinais eletromiográficos, foram utilizados eletrodos de superfície diferenciais simples (DataHominis Tecnologia Ltda., Uberlândia, MG, Brasil) constituído por duas barras retangulares paralelas de prata pura de $10 \mathrm{~mm}$ de comprimento, $1 \mathrm{~mm}$ de largura e distância entre as barras de $10 \mathrm{~mm}$, com circuito pré-amplificador com ganho de 20 vezes, razão de rejeição em modo comum de $92 \mathrm{~dB}$ e razão sinal/ruído $<3 \mu \mathrm{V}$ RMS.

A preparação dos voluntários consistiu em tricotomia e limpeza da pele com álcool 70\%. Os eletrodos de superfície foram colocados nos músculos do antímero direito [1], com sua orientação paralela e as barras de detecção do sinal perpendicular ao sentido das fibras musculares.

Os sinais EMG foram registrados dos seguintes músculos e locais: reto do abdome (RA), alinhado verticalmente e fixado no centro do ventre muscular no ponto médio entre o processo xifóide do osso esterno e cicatriz umbilical, aproximadamente três centímetros lateral e cinco centímetros superior a cicatriz umbilical; oblíquo externo do abdome (OE), superior a espinha iliacaântero-superior (EIAS) do osso do quadril, aproximadamente a 15 centímetros laterais da cicatriz umbilical; oblíquo interno do abdome (OI), alinhado horizontalmente e colocado dois centímetros inferior e medial a EIAS do osso do quadril, localizado no interior de um triângulo delimitado pelo ligamento inguinal, borda lateral da bainha do reto e uma linha que conecta ambas EIAS; multífido (MU), nível do processo espinhoso da quinta vértebra lombar cerca de 2 a 3 centímetros da linha mediana do corpo; eretor da espinha (EE) lateral ao processo espinhoso da terceira vértebra lombar numa distância de aproximadamente 2 a 3 centímetros [2].

Após a colocação dos eletrodos, os voluntários realizaram movimentos específicos de acordo com as funções musculares, para verificar o posicionamento correto e examinar a qualidade do sinal EMG. O eletrodo de referência (Bio-logic Systems - SP Médica, Científica e Comercial Ltda., São Paulo, SP, Brasil), constituído por um disco de aço inoxidável $(30 \mathrm{~mm}$ de diâmetro $\times 1,5 \mathrm{~mm}$ de espessura), foi fixado na pele sobre a crista ilíaca esquerda.

Procedimento experimental: A coleta de dados foi dividida em dois dias distintos. No primeiro, os voluntários passaram por avaliação física e realizaram a familiarização dos exercícios e da contração isométrica voluntária máxima (CIVM). A coleta dos dados eletromiográficos ocorreu uma semana depois [5], no qual foram realizadas duas CIVM de 5 segundos com período de descanso de três minutos, para posterior normalização dos dados. Após o término das CIVM, foram utilizados cinco minutos de intervalo e, posteriormente, os sujeitos realizaram todos os exercícios de estabilização. A ordem destes foi aleatória por meio de sorteio. Cada sujeito executou duas repetições de cada exercício com cinco segundos de contração isométrica, com intervalo de 30 segundos entre as repetições e um minuto entre os diferentes exercícios.

\section{Exercícios executados:}

1- Prancha Lateral (PL): os voluntários ficaram em decúbito lateral com braço direito abduzido a $90^{\circ}$, antebraço direito flexionado a $90^{\circ}$ e cotovelo e antebraço direito apoiados no solo.

2- Prancha Ventral (PV): os voluntários ficaram em decúbito ventral com braços e antebraços flexionados a $90^{\circ}$ e cotovelos e antebraços apoiados no chão.

3- Ponte (PO): o voluntário ficará em decúbito dorsal, com braços estendidos sobre o solo, pernas flexionadas e com os pés apoiados no solo. O quadril e o tronco deverão ser elevados, mantendo uma linha reta entre esses segmentos, e um ângulo de $90^{\circ}$ nos joelhos.

4- Balanço para trás (BA): o voluntário ficará ajoelhado com o tronco ereto e com os joelhos alinhados com o quadril e braços ao longo do corpo. Em seguida, inclinou o tronco para trás, até formar um ângulo de $30^{\circ}$.

5- Prancha ventral de dois apoios direito (2A-D): o voluntário ficou em posição de quatro apoios, em seguida realizou uma elevação do braço direito e extensão da perna esquerda.

6- Prancha ventral de dois apoios esquerdo (2A-E): o voluntário ficou em posição de quatro apoios, em seguida realizou uma elevação do braço esquerdo e extensão da perna direita.

7- Prancha dorsal (PD): o voluntário ficou sentado com os membros inferiores estendidos, tronco levemente inclinado para trás e com as duas mãos no chão. Eles foram instruídos a elevarem o corpo (tronco, quadril e joelhos) formando uma linha reta entre estes segmentos, utilizando como apoio somente os calcanhares a as mãos.

Para análise do sinal, os valores brutos de RMS de cada músculo foram processados e analisados utilizando-se o software Myosystem brl (versão 3.5.6) a partir de uma janela de tempo de 3 segundos centrais, 
correspondendo ao trecho médio da atividade eletromiográfica. Os valores de RMS de cada músculo foram calculados a partir da média das duas repetições em cada exercício e normalizados em termos de porcentagem do valor máximo de RMS obtido das duas sequências de CIVM.

Os testes de CIVM dos músculos do core foram realizados pelos voluntários de acordo com as respectivas funções musculares, conforme as recomendações da literatura específica [2].

A análise estatística dos dados foi realizada utilizando-se o programa computadorizado GraphPadPrism (versão 3.0 - Graphpad Software, Inc). Utilizou-se o teste Kolmogorov-Smirnov para avaliar a normalidade dos dados e, posteriormente, a análise de variância de medidas repetidas (ANOVA) foi utilizada para a comparação das médias dos valores de RMS normalizado (RMSn) do mesmo músculo em diferentes exercícios, sendo que em todas as análises foi realizado o teste de comparações múltiplas Bonferroni para verificar onde havia diferença. O nível de significância foi estabelecido em $5 \%(p<0,05)$.

\section{Resultados}

A atividade eletromiográfica do músculo RA foi maior nos exercícios balanço $(20,99 \% \pm 22,02)$ e prancha ventral $(13,95 \% \pm 12,40)$ em comparação aos exercícios prancha dorsal $(1,66 \% \pm 1,26 ; \mathrm{p}<0,05)$, prancha ventral de dois apoios direito $(1,55 \% \pm 1,16 ; \mathrm{p}<0,05)$, esquerdo $(1,63 \% \pm 1,21 ; \mathrm{p}<0,05)$ e ponte $(1,05 \% \pm 0,88 ; \mathrm{p}<0,05)$. A atividade do RA foi mais alta no exercício balanço em comparação com a prancha lateral $(7,37 \% \pm 6,32$; $\mathrm{p}<0,05)$.

O sinal eletromiográfico (EMG) do músculo $\mathrm{OE}$ foi maior nos exercícios prancha lateral $(27,44 \% \pm 9,44)$, prancha ventral $(24,12 \% \pm 12,49)$ e balanço $(23,43 \%$ $\pm 14,97)$ em comparação aos exercícios prancha dorsal $(4,22 \% \pm 1,28 ; \mathrm{p}<0,05)$, prancha ventral de dois apoios direito $(5,64 \% \pm 3,53 ; \mathrm{p}<0,05)$, esquerdo $(14,38 \% \pm 5,06$; $\mathrm{p}<0,05)$ e ponte $(3,19 \% \pm 3,55 ; \mathrm{p}<0,05)$.

A atividade do mesmo músculo foi maior no exercício prancha ventral de dois apoios esquerdo $(14,38 \% \pm 5,06 ; p<0,05)$ em relação aos exercícios prancha dorsal, prancha ventral de dois apoios direito e ponte.

A atividade eletromiográfica do músculo OI foi maior no exercício prancha lateral $(24,17 \% \pm 15,92$; $\mathrm{p}<0,05)$ em comparação aos outros exercícios (PV: 11,21\% $\pm 4,85 ;$ PD: 7,34\% \pm 7,02; 2A-E: 5,56\% $\pm 3,97$; 2 A-D: $5,31 \% \pm 3,04$; $\quad$ PO: $2,73 \% \pm 1,86$; BA: $15,42 \% \pm 10,16)$.

A atividade do mesmo músculo foi mais alta $(p<0,05)$ no exercício balanço em relação aos exercícios prancha dorsal, prancha ventral de dois apoios (direito e esquerdo) e ponte. O sinal EMG do músculo OI foi maior $(p<0,05)$ no exercício prancha ventral em comparação a ponte.

A atividade eletromiográfica do EE foi mais alta no exercício prancha dorsal $(43,90 \% \pm 15,98)$ em comparação aos outros exercícios (PL: 26,04\% $\pm 10,71$; PV: $2,59 \% \pm 1,34$; 2A-E: $27,96 \% \pm 12,28$; 2 A-D: $28,80 \%$ $\pm 10,25$; PO: $26,02 \% \pm 9,75$; BA: 4,71\% $0 \pm 4,21 ; \mathrm{p}<0,05)$.

$\mathrm{O}$ sinal EMG do mesmo músculo foi maior $(\mathrm{p}<0,05)$ nos exercícios prancha lateral, ponte, prancha ventral de dois apoios direito e esquerdo em relação aos exercícios prancha ventral e balanço.

A atividade eletromiográfica do MU foi maior no exercício prancha dorsal $(37,79 \% \pm 20,69) \mathrm{em}$ comparação a maioria dos outros exercícios (PV: 4,24\% $\pm 3,89$; 2A-D: $23,41 \% \pm 11,75$; PO: $26,35 \% \pm 13,46$; BA: $7,53 \% \pm 6,94 ; \mathrm{p}<0,05)$.

$\mathrm{O}$ sinal EMG do MU foi mais alto nos exercícios prancha lateral $(28,35 \% \pm 14,46)$, prancha ventral de dois apoios direito $(23,41 \% \pm 11,75)$, esquerdo $(29,49 \%$ $\pm 16,38)$ e ponte $(26,35 \% \pm 13,46)$ em relação aos exercícios prancha ventral $(4,24 \% \pm 3,89 ; \mathrm{p}<0,05)$ e balanço $(7,53 \% \pm 6,94 ; p<0,05)$.

\section{Discussão}

No presente estudo analisou-se quantitativamente o recrutamento dos músculos do core durante sete exercícios isométricos de estabilização do tronco, no qual foi demonstrado que a utilização de diferentes exercícios com variações de movimentos em relação aos planos e eixos de movimento, proporcionam respostas distintas no recrutamento dos músculos do core. A compreensão dessas respostas é importante para profissionais da área da saúde determinarem a progressão apropriada nos programas de prevenção e reabilitação do core, ou treinamento esportivo.

Os padrões de ativação muscular registrados durante a realização dos exercícios de estabilização do tronco caracterizou-se pela ativação preferencial dos músculos que atuam contra a gravidade, ou seja, aqueles que controlam o peso da parte inferior do corpo mantendo a coluna vertebral em posição neutra. No presente estudo, ficou demonstrado que os exercícios prancha ventral (PV), prancha lateral (PL) e balanço pra trás (BA) recrutaram com maior magnitude os músculos abdominais (RA, OE e OI), com pequenas variações nos seus valores de ativação.

Estes resultados estão de acordo com os achados de [2], os quais demonstraram maior atividade dos músculos abdominais na prancha ventral e dos músculos oblíquos na prancha lateral. Todavia, deve-se ressaltar que a maior ativação de músculos específicos não significa necessariamente a inatividade de outros músculos estabilizadores, o que ressalta a importância de futuros estudos analisarem os níveis de co-contração durante estes exercícios.

Em relação ao exercício balanço pra trás, nota-se maior ativação do músculo RA em comparação com a maioria dos outros exercícios, porém, não existe nenhum estudo eletromiográfico que avaliou o padrão de ativação dos músculos do core durante este exercício, tornando-se difícil a comparação desses dados.

Este padrão de recrutamento no exercício balanço ocorreu provavelmente devido ao torque gerado com a hiperextensão do tronco, ou seja, este momento de torque poderia ocasionar a queda do voluntario para trás, todavia, este fato não ocorreu porque os músculos abdominais produzem flexão do tronco contra a 
gravidade ou contra uma resistência [6]. Dessa maneira, acredita-se que a ativação do RA gerou uma força de módulo igual e sentido oposto, o que resultou em um equilíbrio das forças, anulando o torque gerado pela hiperextensão do tronco e resultando num exercício isométrico.

A atividade do $\operatorname{EE~}(49,58 \%)$ e $\mathrm{MU}(41,06 \%)$ foi mais alta no exercício prancha dorsal em comparação aos outros exercícios, demonstrando maior recrutamento da musculatura que atua contra a gravidade, como descrito anteriormente. Além disso, o sinal EMG destes músculos (EE e MU) teve maior magnitude nos exercícios ponte, prancha lateral e prancha ventral de dois apoios em comparação a prancha ventral e balanço.

Desta forma, dependendo da posição do corpo durante os exercícios de estabilização (decúbito ventral, lateral ou dorsal), nota-se que o padrão de recrutamento dos músculos do core sofre variações alterando as contribuições relativas de cada músculo necessário para manter a postura e estabilidade da coluna, fatores importantes para a prescrição de exercícios em programas de prevenção e reabilitação ou treinamento esportivo.

Todos os exercícios analisados proporcionaram recrutamento menor de $60 \%$ para os músculos $\mathrm{RA}, \mathrm{OE}$, OI, MU e EE. De acordo com a classificação do nível de atividade muscular [5] em baixa ( $0 \%$ a $20 \%$ CIVM), moderada $(21 \%$ a $40 \%)$, alta $(41 \%$ a $60 \%)$ e muito alta (acima de 60\%), estes exercícios são direcionadas principalmente para a melhora da resistência muscular e controle neuromuscular do tronco.

A resistência muscular é uma qualidade física que deve ser enfatizada no programa de estabilização do tronco, pois, segundo [1] durante o cotidiano, a estabilização da região lombar-pélvica deve ser mantida durante várias horas com a utilização de níveis moderados de atividade muscular, fator que ressalta a importância desses exercícios em um programa de treinamento do core.

Além disso, a utilização de diferentes exercícios de estabilização pode ser um fator importante para que possa ser respeitado o princípio da individualidade do treinamento desportivo, no qual preconiza que o treinamento deve ser elaborado de maneira individualizada para melhor obtenção dos resultados, respeitando as limitações funcionais de cada sujeito [7].

No presente estudo foi utilizado um grupo de jovens, saudáveis, fisicamente ativos e sem históricos de lombalgia, portanto, os resultados devem ser aplicados com precauções para diferentes populações, o que se torna um fator limitante deste trabalho. Outra limitação foi à ausência da avaliação da atividade elétrica bilateral dos músculos do tronco, fator importante para compreender o padrão de ativação dos músculos do core em diferentes exercícios de estabilização.

\section{Conclusão}

Os músculos abdominais (RA, OE e OI) foram recrutados com maior magnitude nos exercícios prancha ventral, prancha lateral e balanço pra trás, enquanto os músculos extensores do tronco (EE e MU) produziram maior ativação eletromiográfica nos exercícios ponte, prancha dorsal e prancha ventral de dois apoios, desta forma, os padrões de ativação muscular registrados durante a realização da maioria dos exercícios de estabilização, caracterizou-se pela ativação preferencial dos músculos que atuam contra a gravidade.

A prancha lateral pode ser considerada um exercício importante e interessante para melhorar a estabilidade da coluna devido a maior ativação dos músculos agonistas e antagonistas do tronco em comparação aos outros exercícios.

Os exercícios de estabilização do tronco podem ser utilizados para diversificar um programa de treinamento d o core e são particularmente adequados para o desenvolvimento da resistência muscular e melhorar a capacidade de estabilização da coluna vertebral.

\section{Referências}

[1] Tan S, Cao L, Schoenfisch W, Wang J. Investigation of Core Muscle Function through Electromyography Activities in Healthy Young Men. Journal of Exercise Physiology. 2013;16(1): 45-52.

[2] García-Vaquero MP, Moreside JM, Brontons-Gil E, Peco-González N, Vera-Garcia FJ. Trunk muscle activation during stabilization exercises with single and double leg support. Journal of electromyography and kinesiology. 2012;22:398-406.

[3] Basmajian JV, De Luca CJ. Muscles alive: their function revealed by electromyography. 5. ed. Baltimore: Williams and Wilkins; 1985.

[4] Marshall P W, Desai I. Electromyographic analysis of upper body, lower body, and abdominal muscles during advanced Swiss ball exercises. Journal of Strength and Conditioning Research. 2010;24(6):1537-45.

[5] Escamilla RF, Lewis C, Bell D, Bramblet G, Daffron $\mathrm{J}$, Lambert $\mathrm{S}$ et al. Core muscle activation during Swiss ball and traditional abdominal exercises. Journal of Orthopaedic \& Sports Physical Therapy. 2010; 40(5):265-76.

[6] Hamill J, Knutzen KM. Bases biomecânicas do movimento humano. $3^{\mathrm{a}}$ ed. São Paulo: Manole; 2012.

[7] B ompa TO.A periodização no treinamento esportivo. São Paulo: Manole; 2001. 